\title{
OPTOGENETICA: CONTROLLARE I NEURONI CON LA LUCE
}

\author{
ANNA MORONI (*) \\ Nota presentata dal s.c. Martino Bolognesi \\ (Adunanza del 22 gennaio 2015)
}

SunTO. - L'optogenetica è una tecnica in forte espansione nelle neuroscienze che permette di manipolare l'attività neuronale con la luce. Abbiamo creato mediante tecniche di ingegneria proteica un canale ionico selettivo al potassio $\left(\mathrm{K}^{+}\right)$che permette di inibire l'attività elettrica dei neuroni con la luce. Il canale BLINK è stato ottenuto collegando il modulo LOV2 della proteina fototropina di pianta al canale ionico virale Kcv. In seguito a illuminazione con luce blu (450 nm), BLINK iperpolarizza il potenziale di membrana dei neuroni e ne impedisce il firing. Esperimenti pilota in un modello murino di dolore neuropatico hanno mostrano che BLINK può essere impiegato per ridurre la sensazione di dolore a lungo termine.

$* * *$

ABSTRACT. - Optogenetics is a booming technique in neuroscience that allows manipualtion of neuronal activity by light. Neuronal activation can be achieved by the activation of light-gated cation channels while neuronal inhibition relies on the activation of chloride pumps and channels. We have engineered a light-gated potassium $\left(\mathrm{K}^{+}\right)$channel (BLINK) by linking the plant photoreceptor module LOV2 to the viral channel Kcv. Upon illumination with blue light (450 nm), BLINK channel hyperpolarizes neurons to $\mathrm{E}_{\mathrm{K}}$ and this effect persists for several minutes even in the dark. Pilot experiments in a mouse pain model show that this protracted light-off activity can be successfully employed for long term pain relief after activation of the channel by a short pulse of light.

(") Dipartimento di Bioscienze, Università degli Studi di Milano, Italia.

E-mail: anna.moroni@unimi.it 


\section{INTRODUZIONE}

Da anni molte aree della biologia e della medicina sono alla ricerca di un sistema non invasivo che permetta di controllare le funzioni delle cellule con la luce [1]. Un progresso notevole in questo campo è stato raggiunto recentemente dall'optogenetica, una tecnologia che utilizza proteine geneticamente codificate sensibili alla luce per controllare un ampio spettro di bersagli che vanno dalle singole proteine alle complesse cascate di trasduzione del segnale [2]. Un'applicazione frequente dell'optogenetica è nell'ambito della neurobiologia, dove la luce viene utilizzata per controllare l'attività dei neuroni o di altre cellule eccitabili che esprimono proteine fotoattivabili [3]. La stimolazione a distanza dei circuiti neuronali è comunemente ottenuta attraverso canali cationici regolati dalla luce, channelrodopsin $(\mathrm{ChR})$ che depolarizzano la cellula [4]. L'inibizione invece è ottenuta principalmente attraverso pompe ioniche fotosensibili, che trasportano ioni e iperpolarizzano le cellule. Le pompe come batterio-rhodopsina e alorhodopsina, utilizzano a questo scopo l'energia luminosa per spostare protoni $\left(\mathrm{H}^{+}\right)$o ioni cloruro $\left(\mathrm{Cl}^{-}\right)$contro i loro gradienti [5]. Sebbene in linea di principio siano adatte per iperpolarizzare le cellule e per controllarne l'eccitabilità, le pompe ioniche a base di opsina sono piuttosto inefficienti come strumenti optogenetici perché muovendo un solo ione per fotociclo, non riducono in maniera rapida la resistenza d'ingresso delle membrane cellulari. Inoltre, il funzionamento delle pompe crea gradienti ionici anomali nella cellula. Uno strumento alternativo per la riduzione efficiente delle correnti eccitatorie è stato ingegnerizzato trasformando le ChR in canali selettivi per lo ione cloro [6,7]. Questi canali si sono dimostrati efficaci per iperpolarizzare le cellule ma il loro uso è purtroppo limitato ad una certa classe di neuroni, cioè quelli con un gradiente favorevole per l'ingresso di $\mathrm{Cl}^{-}$[8]. Un'altra limitazione all'uso di questi canali è la loro stretta dipendenza dal $\mathrm{pH}$. A causa di queste carenze, sia delle pompe che dei canali di $\mathrm{Cl}^{-}$, la comunità optogenetica è ancora alla ricerca di un canale più efficiente e che possa essere utilizzato per l'iperpolarizzazione di tutti i tipi di cellule. Dato il ruolo universale dei canali per il $\mathrm{K}^{+}$nel terminare i potenziali d'azione e prevenire l'attività di firing delle cellule eccitabili [9], un canale per il $\mathrm{K}^{+}$controllato dalla luce sarebbe lo strumento optogenetico ideale per l'inibizione neuronale. Diversi laboratori hanno affrontato questo problema e progettato allo scopo canali per il $\mathrm{K}^{+}$sintetici e luce-attivati. Ad esem- 
pio, è stato ingegnerizzato il canale SPARK, legando ad esso una molecola di azobenzene collegata ad uno ione tetraetilammonio che blocca il poro. L'assorbimento della luce, determina la contrazione dell'azobenzene che rimuove il bloccante dal poro attivando il canale $[10,11]$. Altri gruppi hanno invece utilizzato aminoacidi non naturali sensibili alla luce inseriti nelle proteine canale per indurne un gating foto-attivabile [12]. Infine, si è provato a coesprimere il canale con una proteina che funziona da interruttore fotoattivabile (lumitossina) che rilascia in prossimità del canale una tossina inibitoria [13].

Tutti questi canali sono in grado d'iperpolarizzare le cellule e inibire il firing neuronale. Tuttavia, ciascuno di essi presenta alcuni svantaggi, che ne limitano l'applicazione. Il canale SPARK, ad esempio, richiede l'aggiunta di azobenzene come co-fattore esogeno [10,11]; questo può limitarne l'applicazione. Il canale che funziona con la lumitossina è già attivo anche al buio e ha pertanto una gamma dinamica molto ristretta, il che rende questo costrutto piuttosto inefficace [13]. Infine, l'attivazione da luce di un aminoacido non naturale inserito nel canale non è reversibile [12].

Nel complesso, questa breve panoramica degli strumenti optogenetici utilizzati per l'iperpolarizzazione delle cellule sottolinea che il campo è ancora alla ricerca di un canale $\mathrm{K}^{+}$completamente codificato geneticamente, attivato in modo reversibile e perfettamenete chiuso al buio.

\section{RISULTATI}

Ispirati dalla necessità di un canale $\mathrm{K}^{+}$con le suddette caratteristiche, abbiamo recentemente ingegnerizzato un canale sintetico, in cui un modulo "sensore", il dominio LOV2 della fototropina vegetale, è stato collegato al canale per il $\mathrm{K}^{+} \mathrm{Kcv}$, che funge da modulo "effettore". Il design di questo canale sintetico è stato ispirato da risultati precedenti in cui avevamo dimostrato che i canali del $\mathrm{K}^{+}$sono elementi modulari in cui il poro, che conduce gli ioni, è collegato ai domini regolatori, che ne controllano l'apertura e la chiusura [14]. Qualsiasi modifica conformazionale del dominio regolatorio viene trasmessa all'unità del poro, alterando il flusso ionico. Questa architettura naturale dei canali di $\mathrm{K}^{+}$ può essere imitata in proteine sintetiche accoppiando al poro del canale un "sensore" esogeno, prelevato cioè da altre proteine [14]. Con questa logica avevamo costruito in passato, canali ionici sintetici attivati da vari 
stimoli: voltaggio, calcio intracellulare e ROS $[14,15,16]$. L'elemento centrale di questi canali è un poro selettivo $\mathrm{al} \mathrm{K}^{+}$scoperto in precedenza in una classe di virus, Phycodnaviridae, che infetta le alghe [17]. I canali virali sono strutturalmente semplici e funzionalmente molto robusti, mantenendo la loro funzione anche in seguito a significative modifiche strutturali. I canali virali rappresentano pertanto un elemento essenziale per il successo dei progetti d'ingegneria $[14,15,16]$. Tale successo è in parte dovuto alla conoscenza dettagliata del loro funzionamento. Negli ultimi dieci anni, nel nostro laboratorio si è studiato a fondo la relazione struttura-funzione di queste piccole proteine $[17,18]$ arrivando a comprenderne i cambiamenti conformazionali alla base del meccnismo di aperutra e chiusura [19].

Il progetto d'ingegneria proteica più impegnativo è stata la costruzione ex novo di un canale $\mathrm{K}^{+}$sensibile alla luce blu. In questo progetto abbiamo collegato il poro del canale virale $\mathrm{Kcv}$ al dominio LOV2 (Luce - Ossigeno- Voltaggio) dalla fototropina, un recettore della luce blu di pianta. La fusione delle due componenti proteiche ha richiesto diverse fasi di ottimizzazione, che sono state effettuate sia in lievito con l'ausilio della tecnica della complementazione di mutanti incapaci di assorbire $\mathrm{K}^{+}$, sia misurando le correnti ioniche in sistemi eterologhi con la tecnica del patch clamp. La Fig. 1 mostra lo schema generale del canale che abbiamo chiamato BLINK-1, e l'evidenza sperimentale dell'attivazione/deattivazione, reversibile del canale in risposta alle transizioni luce/buio in una cellula di mammifero. La sensibilità alla luce blu del canale è dovuta al cromoforo flavina mononucleotide, FMN, associata al dominio LOV2. In seguito all'assorbimento di luce blu, la flavina si lega in modo covalente alla proteina che subisce un cambiamento conformazionale favorendo il movimento di un'alfa elica $(\mathrm{J} \alpha)$. Questo cambiamento conformazionale indotto dalla luce nel dominio LOV2 era già stato utilizzato da altri come componente costruttivo di proteine optogenetiche sintetiche [20]. La descrizione dettagliata delle proprietà di base del canale BLINK-1 attivato dalla luce blu è riportata nel lavoro pubblicato nel 2015 sulla rivista Science [21]. I dati evidenziano alcune caratteristiche funzionali positive di questo canale sintetico:

i) è completamente codificato geneticamente,

ii) è chiuso al buio e si attiva in risposta all'illuminazione con luce blu,

iii) non si disattiva durante illuminazione prolungata,

iv) l'attivazione del canale è completamente reversibile al buio, 
v) l'attivazione è molto sensibile alla luce e la metà dell'attivazione massima si ottiene con $25 \mu \mathrm{W} / \mathrm{mm}^{2}$,

vi) la conduttanza unitaria è elevata $>100 \mathrm{pS}$,

vii) il canale può essere espresso in zebrafish e utilizzato in vivo per la modulazione del comportamento dei pesci.
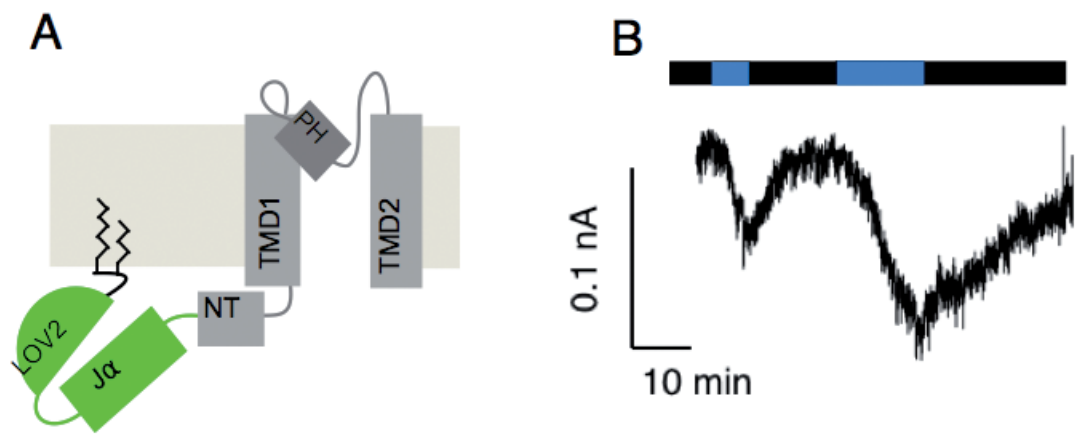

Fig. 1 - Schema costruttivo e funzione del canale per il $K^{+}$sensibile alla luce blu BLINK-1. (A) Ogni monomero comprende il canale virale Kcv (grigio) con domini transmembrana (TMD1, TMD2), elica del poro (PH) e N-terminale citosolico (NT), il dominio LOV2 (verde) dalla fototropina, l'elica Ja e un dominio di miristilazione (nero).

(B) Quando il canale è espresso transientemente in cellule HEK293 di mammifero, il passaggio da buio a luce blu determina l'apertura del canale con un conseguente aumento della conduttanza della cellula al $\mathrm{K}^{+}$, che si inverte al buio (da Cosentino et al., 2015).

Ulteriori applicazioni del canale BLINK1 hanno rivela- to alcuni difetti di questa proteina sintetica. Uno dei limiti rilevati è la sua bassa espressione nelle cellule neuronali di mammifero, limite che ha impedito l'applicazione del nuovo strumento optogenetico in modelli animali quali topo e ratto. Uno degli obiettivi principali delle successive modifiche alla proteina BLINK-1 è stato migliorarne l' espressione in neuroni di topo. Dopo aver provato diversi segnali di trafficking cellulare, abbiamo ottenuto un nuovo canale chiamato BLINK2, che ha dato risultati positive in mammifero [22]. I principali risultati riassunti in breve sono:

- BLINK2 si esprime in neuroni di mammifero (topo e ratto). In collaborazione con Monica Di Luca, (Università degli Studi di Milano) abbiamo infettato le colture neuronali ippocampali primarie di ratto con un costrutto virale (AAV) che esprime BLINK2 sotto il controllo del promotore umano Synapsin (hSyn) (AAV - hSyn- 
BLINK2). L'analisi delle immagini confocali mostra che BLINK2 è espresso sulla superficie dei neuroni ed è principalmente localizzato nel distretto somatodendritico.

- BLINK2 può essere usato per silenziare i neuroni. Il canale è stato espresso da Raffaella Tonini (IIT, Genova) nel nucleo dorsale del rafe (DRN) del mesencefalo di topo. Registrazioni elettriche su fettina hanno mostrato che una breve esposizione alla luce blu induce una rapida iperpolarizzazione della membrana e un completo silenziamento dell'attività elettrica del neurone. I dati mostrano inoltre che questa inibizione è di lunga durata e che il recupero al buio è molto lento (dell'ordine delle decine di minuti).

- L'attivazione di BLINK2 altera il comportamento nei modelli animali. In una linea transgenica stabile di zebrafish, che esprime BLINK2, abbiamo ottenuto l'espressione mirata di BLINK2 in motoneuroni e l'alterazione della risposta di fuga degli embrioni. Inoltre, BLINK2 è stato provato anche in ratto. Studi comportamentali hanno dimostrato che un'attivazione di BLINK2 nei neuroni sensoriali riduce notevolmente la sensazione di dolore dell'animale. Questo ci permette di ipotizzare un impiego futuro di BLINK2 nella terapia del dolore periferico neuropatico.

\section{BIBLIOGRAFIA}

[1] Tischer, D., Weiner, O.D. (2014) Illuminating cell signaling with optogenetic tools. Nat. Rev. Mol. Cell Biol. 15:551-558.

[2] Zhou, X.X. et al. (2015) Investigating neuronal function with optically controllable proteins. Frant. Mol. Neurosci. 8:1-15.

[3] Fenno, L., Yizahar, O., Deisseroth, K. (2011) The development and application of optogenetics. Annu. Rev. Neurosci. 34:389-412.

[4] Nagel, G. et al. (2005) Channelrhodopsins direct light-gated cation channels. Biochem. Soc. Trans. 33:863-866.

[5] Kandori, H. (2015) Ion-pumping microbial rhodopsins. Front Mol. Biosci. 52:doi 10.3389/fmolb.2015.00052.

[6] Berndt, A. et al. (2014) Structure-guided transformation of channelrhodopsin into a light-activated chloride channel. Science 344:420-424.

[7] Wietek, J. et al. (2014) Conversion of channelrhodopsin into a light-gated chloride channel. Science 344:420-424.

[8] Hübner, C. et al (2001) Disruption of KCC2 reveals an essential role of K-Cl cotransport already in early synaptic inhibition. Neuron 30:515-524. 
[9] Hille, B. (2001) Ionic channels of excitable membranes. Sinauer Sunderland MA.

[10] Banghart, M., et al. (2004) Light-activated ion channels for remote control of neuronal firing. Nature Neuroscience 7:1381-1386.

[11] Janovjak, H. et al. (2010) A light-gated, potassium-selective glutamate receptor for the optical inhibition of neuronal firing. Nat. Neuroscience 13:1027-1032.

[12] Kang, J.Y. et al. (2013) In vivo expression of a light-activatable potassium channel using unnatural amino acids. Neurone 80:358-370.

[13] Schmidt, D. et al. (2013) A fully genetically encoded protein architecture for optical control of peptide ligand concentration. Nat. Commun. 5: doi:10.1038/ncomms4019.

[14] Arrigoni, C. et al. (2013) The voltage-sensing domain of a phosphatase gates the pore of a potassium channel. J. Gen. Physiol. 141:389-3895.

[15] DiFrancesco, M.L. et al. (2015) Engineering a $\mathrm{Ca}^{2+}$ sensitive (bio) sensor from the pore-moduke of a potassium channel. Sensors 15:4913-4924.

[16] Schroeder, I. et al. (2013) Creation of a ROS-insensitive Kcv channel. Biochemistry 52:3031-3037.

[17] Plugge, B., et al. (2000). A potassium ion channel protein encoded by chlorella virus PBCV-1. Science 287:1641-1644.

[18] Tayefeh, S. et al. (2009) Model Development for the Viral Kcv Potassium Channel. Biophys. J. 96:485-498.

[19] Hoffgaard, F. et al. (2015) Tectonics of a $\mathrm{K}^{+}$channel: The importance of the Nterminus for channel gating. Biochim. Biophys. Acta 1848:3197-3204.

[20] Wu, Y.I. et al. (2009) A genetically encoded photoactivatable Rac controls the motility of living cells. Nature 461:104-108.

[21] Cosentino, C. et al. (2015) Engineering of a light-gated potassium channel. Science 348:707-710.

[22] Alberio, L. et al. (2018) A light-gated potassium channel for sustained neuronal inhibition. Nature Methods 15:969-976. 
